\title{
Five-Year Evaluation of the Urine Culture Results and Antimicrobial Susceptibility Profiles of Isolated $E$. coli Strains
}

\author{
Osman Aktaş* and Elif Denktaş \\ Department of Medical Microbiology, Turkey \\ *Corresponding author: Osman Aktas, Department of Medical Microbiology, Faculty of Medicine, Prof. Dr. Ataturk University, \\ Erzurum, Turkey
}

\section{ARTICLE INFO}

Received: 幽 December 18, 2019

Published: 幽January 06, 2020

Citation: Osman Aktaş, Elif Denktaş. FiveYear Evaluation of the Urine Culture Results and Antimicrobial Susceptibility Profiles of Isolated E. coli Strains. Biomed J Sci \& Tech Res 24(2)-2020. BJSTR. MS.ID.004029.

Keywords: E. Coli; Urine Culture; Urinary Tract Infections; Antimicrobial Resistance; Turkey

Abbreviations: UTI: Urinary tract infection; CLSI: Clinical and Laboratory Standards Institute; EUCAST: European Committee on Antimicrobial Susceptibility Testing; AMK: amikacin; AMP: Ampicillin; SAM: Ampicillin/Sulbactam; CPM: Cefepime; CTX: Cefotaxime; CAZ: Ceftazidime; CRO: Ceftriaxone; CXM: Cefuroxime; CIP: Ciprofloxacin; ETP: Ertapenem; GEN: Gentamicin; IMP: Imipenem; MPM: Meropenem; PIP: Piperacillin; TZP: Piperacillin/Tazobactam; SXT: Trimethoprim/Sulfamethoxazole; AZT: Aztreonam; ESBL: Extended-Spectrum B-Lactamase

\section{ABSTRACT}

Background/Aim: The objectives of this study was to evaluate the microbiological populations in urine cultures and to investigate the variations in antimicrobial resistance profiles of urinary Escherichia coli strains.

Materials and Methods: Results of 162,083 urine cultures were evaluated retrospectively. Diagnosis of isolated microorganisms and antibiotic susceptibility of Escherichia coli strains were investigated by routine methods or automated identification and susceptibility test systems [VITEK® 2 (bioMérieux Clinical Diagnostics) or Phoenix ${ }^{\mathrm{TM}}$ [Becton Dickinson Diagnostic Systems].

Result: Positive results were obtained from $15.8 \%$ of the cultured specimens; contamination was observed in $10.5 \%$. Culture positivity was greater in women than in men and was higher in early ages and in older people than in younger people. Culture positivity was far more common in outpatients (9.7\%) than in inpatients (6.1\%). The most frequently isolated bacteria were Escherichia coli (48.0\%), followed by Enterococcus spp. (9.0\%), coagulase-negative staphylococci (8.3\%) and Klebsiella spp. (7.6\%). Of the E. coli strains, $27.8 \%$ were extended-spectrum $\beta$-lactamases positive. The most effective antibiotics for $E$. coli strains were imipenem, meropenem, ertapenem and amikacin. Ampicillin/sulbactam, ciprofloxacin and tazobactam resistance in ESBL-producing $E$. coli strains and ampicillin/sulbactam, cefepime, cefotaxime, ceftazidime, ceftriaxone, ciprofloxacin, gentamicin, tazobactam and trimethoprim/sulfamethoxazole resistance in non-ESBL-producing E. coli strains showed a significant increase in 2018.

Conclusion: Community or hospital-acquired urinary tract infection rates were high in Erzurum. E. coli strains are highly resistant to a significant proportion of antibiotics used in treatment. The increase in the antimicrobial resistance of the bacterium is of concern in our region. 


\section{Introduction}

Urine specimens, which can be obtained without invasive procedures, allow the diagnosis of urinary tract infections (UTI).

These specimens provide important clues for diagnosis, even based on color and consistency, and they allow direct searches for bacterial antigens in cases where traditional culture methods are inadequate. UTIs occupy an important place among communityacquired and hospital-acquired bacterial infections. They can cause clinical conditions that range from asymptomatic bacteriuria to urethritis, cystitis, prostatitis, pyelonephritis, and sepsis [1]. UTIs that threaten human health with antibiotic-resistant uropathogens affect more than 150 million people each year in both developed and developing countries, and they are financially damaging to a country's economy [2]. Women, infants, the elderly, and patients hospitalized and undergoing surgery or invasive procedures are high-risk groups for UTIs [3-5]. UTIs are second only to pneumonia as the most common cause of hospitalization, and failure or delays in treatment can give rise to a mortality of $20-50 \%$ [6].

The most common pathogens observed in UTIs are Escherichia coli (E. coli), Klebsiella pneumoniae, Pseudomonas aeruginosa, and Staphylococcus, Enterococcus, Proteus, and Candida species [7]. High recurrence rates and increased antimicrobial resistance of uropathogens after UTIs pose significant threats to national economies [8]. Preventing the increase of bacterial resistance to antibiotics requires that physicians assign treatments according to the results of antibiograms, rather than empirically, unless the latter is mandatory. The aim of this retrospective study was to obtain microbiological data about the status of UTI in our region (Erzurum, Turkey), to determine the antimicrobial resistance profiles of $E$. coli strains known to be the most frequent causes of UTI, and to contribute to the development of clinical strategies for the prevention and management of these infections.

\section{Materials and Methods}

The urine specimens sent to Erzurum Atatürk University Research and Application Centre Medical Microbiology Laboratory within the five-year period from the beginning of January 2014 to the end of December 2018 were evaluated retrospectively. Patient information and laboratory data were transferred to computer using the Java-based "ENLİL Hospital Information Management System Modules" program. Urine specimens were collected over a $24 \mathrm{~h}$ period. Samples taken from containers with leaks or with Foley catheter tips or from urine collection bags from patients with catheters were not accepted for culture. In total, 213 specimens were submitted incorrectly and were excluded from the assessment. Ultimately, 162,083 urine cultures from 80,524 males and 81,559 females were evaluated in our laboratory.

\section{Collecting and Sending of Urine Specimens}

Clean-catch urine samples from adults, from children who had toilet training, from suprapubic bladder punctures from infants, and from catheter urine from patients with catheterization were collected in standard preservative-free urine tubes. The specimens from the relevant clinics and polyclinics were cultured as soon as they arrived at the laboratory or within one hour at the latest. For specimens containing preservatives, this period was extended up to two hours.

\section{Urine Culture and Bacteriological Media}

A calibrated loop was immersed perpendicular to the urine specimen and approximately $0.01 \mathrm{ml}$ of the urine drop formed on the loop was plated on Eosin Methylene Blue (EMB) agar and Blood Agar plates. The inoculated media were incubated at $35-37^{\circ} \mathrm{C}$ for 18-24 hours.

\section{Evaluation of the Microbial Growth in Cultures}

Colony numbers of microorganisms on the growth-positive plates were counted at the end of incubation. The number of colonies per $1 \mathrm{ml}$ of urine were calculated by multiplying the number of colonies (viable bacteria) on the plate by 100 . At least 10,000 colonies $\left(10^{4} \mathrm{cfu} / \mathrm{mL}\right)$ belonging to a single bacterium were considered. Urine culture results were evaluated according to

a) The probability (low or high) that the specimen was contaminated.

b) The microorganism species diversity isolated in culture.

c) The number of microorganisms that grew in a certain volume of urine. Urine specimens obtained by suprapubic aspirations, surgically placed catheters, and surgical operations were considered to be specimens with a low probability of contamination. Middle urine or urinary or suprapubic permanent catheter urine samples were considered to have a high probability of contamination. In our laboratories, antibiogram procedures are applied only for primary UTI pathogens.

\section{Diagnosis of Microorganisms}

All microorganisms showing growth $\geq 10^{4} \mathrm{cfu} / \mathrm{mL}$ in noncontaminated cultures were identified by routine methods according to Gram staining; colony morphology on the plates; catalase, oxidase and coagulase tests; the growth properties in in triple sugar iron agar slants; and indole, urease, citrate, and motility test results. Automated identification and antibiotic susceptibility test system devices, such as the $\operatorname{VITEK}^{\circledR} 2$ (bioMérieux Clinical Diagnostics, France) and BD Phoenix ${ }^{\mathrm{TM}}$ (Becton Dickinson Diagnostic Systems, France) device, were used to diagnose some bacteria.

\section{Antibiotic Susceptibility Tests}

Antibiotic susceptibility tests were performed either by the Müller Hinton agar disc diffusion technique or by two different automatic identification and sensitivity test system devices. Sensitivity results were evaluated with the VITEK® 2 device (bioMérieux, Durham, NC) according to the Clinical and Laboratory Standards Institute (CLSI) breakpoint criteria and with the BD Phoenix ${ }^{\mathrm{TM}}$ device 
(Becton-Dickinson Diagnostics, Sparks, MD) according to the European Committee on Antimicrobial Susceptibility Testing (EUCAST) breakpoint criteria. The antibiotics used in the antibiotic susceptibility testing were amikacin (AMK), ampicillin (AMP), ampicillin/ sulbactam (SAM), cefepime (CPM), cefotaxime (CTX), ceftazidime (CAZ), ceftriaxone (CRO), cefuroxime (CXM), ciprofloxacin (CIP), ertapenem (ETP), gentamicin (GEN), imipenem (IMP), meropenem (MPM), piperacillin (PIP), piperacillin/tazobactam (TZP), and trimethoprim/sulfamethoxazole (SXT).The CLSI recommendations were followed to establish inhibition zone diameters for sensitivity as CTX $\leq 22 \mathrm{~mm}$, CRO $\leq 19 \mathrm{~mm}, \mathrm{CAZ}$ or aztreonam $\leq 17 \mathrm{~mm}$; these decreases were evaluated as extended-spectrum $\beta$-lactamase

\section{Result}

Table 1: Characteristics of study population and obtained results by gender*.

\begin{tabular}{|c|c|c|c|c|}
\hline Characteristics & Female & Male & All & $\chi^{2} ;$ df $^{* *} ; \mathbf{p}$ value \\
\hline Age range (years) & $0-102$ & $0-100$ & $0-102$ & - \\
\hline Age (mean \pm SD) & $31.1 \pm 24.4$ & $36.9 \pm 27.7$ & $34.0 \pm 26.3$ & - \\
\hline Rejected samples & $103(0.06)$ & $110(0.07)$ & $213(0.1)$ & - \\
\hline Accepted samples & $81,559(50.3)$ & $80,524(49.6)$ & 162,083 (99.9) & - \\
\hline \multicolumn{5}{|c|}{ Contamination } \\
\hline Positive & $11,272(7.0)$ & $5,713(3.5)$ & $16,985(10.5)$ & $1953.92 ; 1 ;<0.0001$ \\
\hline Negative & $70,287(43.4)$ & $74,811(46.2)$ & $145,098(89.5)$ & \\
\hline \multicolumn{5}{|c|}{ Pathogen Growth } \\
\hline Positive & $14,861(9.2)$ & $10,672(6.6)$ & $25,533(15.8)$ & $753.53 ; 1 ;<0.0001$ \\
\hline Negative & $66,698(41.2)$ & $69,852(43.1)$ & $136,550(84.2)$ & \\
\hline \multicolumn{5}{|c|}{ Patients from } \\
\hline Outpatient & $57,354(35.4)$ & $45,458(28.0)$ & $102,812(63.4)$ & $3360.17 ; 1 ;<0.0001$ \\
\hline Inpatient & $24,205(14.9)$ & $35,066(21.6)$ & $59,271(36.6)$ & \\
\hline \multicolumn{5}{|c|}{ UTI prevalence $* * *$} \\
\hline Outpatient & $9,995(6.2)$ & $5,728(3.5)$ & $15,723(9.7)$ & $484.41 ; 1 ;<0.0001$ \\
\hline Inpatient & $4,866(3.0)$ & $4,944(3.1)$ & $9,810(6.1)$ & \\
\hline Total & $14,861(9.2)$ & $10,672(6.6)$ & $25,533(15.8)$ & \\
\hline
\end{tabular}

Note:

* The percentages are given in parentheses.

** $\mathrm{df}=$ Degree of Freedom

*** The percentages were calculated for 162,083 patients.

In the five-year period covering the years 2014 to 2018, a total of 162,296 urine cultures were requested from different clinics and polyclinics of our hospital. In our laboratory, 213 specimens were not accepted because they were not suitable for microbiological examination. The age range of the remaining 162,083 cases included in the study was 0-102 years, with a mean age of $34.0 \pm$ 26.3 years (95\% confidence interval [CI]: 33.9, 34.1).In our hospital, $63.4 \%$ of the urine cultures were obtained from outpatients and $36.6 \%$ from inpatients. A numerical superiority in favor of women in outpatients over inpatients was statistically significant. Of the
(ESBL) positive [9]. According to EUCAST recommendations, ESBL was evaluated as positive in cases with $\geq 5 \mathrm{~mm}$ inhibition zone contraction for both CAZ clavulanate $(30 / 10 \mu \mathrm{g})$ and CTX clavulanate $(30 / 10 \mu \mathrm{g})$ versus CAZ [10].

\section{Statistical Analysis}

The data and laboratory results for the patients were entered into a data file using Microsoft Office 2000 Excel. The ages of the cases were determined as days, and the mean age and standard deviation were determined based on these values. The Chi-square ( $\chi 2)$ test (all two-tailed) was applied to determine the relationship between variables. A value of $\mathrm{p}<0.05$ was considered statistically significant. 
Table 2: Distribution of culture (pathogen) positivity by gender in age groups.

\begin{tabular}{|c|c|c|c|c|c|}
\hline Age in Years & $\mathbf{n}$ & Female, $\mathbf{n}(\mathbf{\%})$ & Male, $\mathbf{n}(\mathbf{\%})$ & All $\mathbf{n}(\%)$ & $\chi^{2} ; \mathbf{d f} ; \mathbf{p ~ v a l u e}$ \\
\hline $0-5$ & 28,988 & $2.610(9.0)$ & $3.503(12.1)$ & $6.113(21.1)$ & $4.587(13.4)$ \\
\hline $6-17$ & 34,293 & $3.612(10.5)$ & $975(2.8)$ & $4.043(10.9)$ & $2452.13 ; 4 ;<0.0001$ \\
\hline $18-44$ & 37,256 & $3.147(8.4)$ & $896(2.4)$ & $4.777(14.1)$ & \\
\hline $45-64$ & 33,968 & $2.768(8.1)$ & $2.009(5.9)$ & $6.013(21.8)$ & \\
\hline$>64$ & 27,578 & $2.724(9.9)$ & $3.289(11.9)$ & \\
\hline
\end{tabular}

The number of urine cultures that exhibited growth $\geq 10^{4}$ CFU per milliliter of urine was 25,553 in the five-year period. The distribution of bacteria isolated from inpatients and outpatients is given in Table 3 , which shows that nearly half $(48.0 \%)$ of the microorganisms were E. coli strains. Enterococcus spp. strains (9.0\%) were the second most common microorganisms, followed by strains of coagulase-negative staphylococci (CNS) (8.3\%), Klebsiella spp. (7.6\%), and Candida spp. (6.9\%). In this study, 15,723 (61.6\%) uropathogens were isolated from outpatients and 9,810 (38.4\%) from inpatients. Staphylococcus aureus positivity did not show statistically significant variability in inpatients and outpatients, whereas the numbers of E. coli, Klebsiella spp., Citrobacter spp., Enterobacter spp., Proteus spp., coagulase-negative staphylococci (CNS), streptococci, and diphtheroids were significantly higher in outpatients than in inpatients, while numbers of Pseudomonas, Acinetobacter, Enterococcus, and Candida spp. were higher in inpatients than in outpatients. Of the 12,247 E. coli strains isolated, 3,399 (27.8\%) were ESBL positive. Table 4 shows the susceptibility of $E$. coli strains to various antibiotics, according to the urine collection year. The most effective antibiotics for ESBL-producing strains were imipenem, meropenem, amikacin, and ertapenem. More than $97 \%$ of the ESBL-positive E. coli strains were resistant to ampicillin and piperacillin; resistance rates to other antibiotics ranged from 29.1-69.3\%. In 2018, ESBL-positive E. coli strains showed a statistically significant increase in resistance to TZP and CIP; a decrease was observed for SAM according to the total of the previous 4 years. No increasing antimicrobial resistance was detected in other ESBL-producing strains in the following years.

Table 3: Prevalence of uropathogens isolated from 25,533 positive cultures.

\begin{tabular}{|c|c|c|c|c|c|c|c|}
\hline \multirow{2}{*}{ Microorganisms } & \multicolumn{2}{|c|}{ Outpatients (n= 15,723) } & \multicolumn{2}{|c|}{ Inpatients (n= 9,810) } & \multicolumn{2}{|c|}{ Total (n = 25,533) } & \multirow{2}{*}{$\begin{array}{c}\chi^{2} ; \text { df; p value Outpatients vs } \\
\text { Inpatients }\end{array}$} \\
\cline { 2 - 7 } & $\mathbf{n}$ & $\mathbf{\%}$ & $\mathbf{n}$ & $\mathbf{\%}$ & $\mathbf{n}$ & $\mathbf{\%}$ & $792.94 ; 1 ;<0.0001$ \\
\hline E. coli & 8.635 & 33.8 & 3.612 & 14.1 & 12,247 & 48 & $310.12 ; 1 ;<0.0001$ \\
\hline Enterococcus spp. & 1,025 & 4 & 1,276 & 5 & 2,301 & 9 & $32.67 ; 1 ;<0.0001$ \\
\hline CNS & 1,421 & 5.6 & 688 & 2.7 & 2,109 & 8.3 & $10.35 ; 1 ; 0.0130$ \\
\hline Klebsiella spp. & 1.135 & 4.4 & 816 & 3.2 & 1,951 & 7.6 & $1828.42 ; 1 ;<0.0001$ \\
\hline Candida spp. & 245 & 1 & 1,523 & 6 & 1,768 & 6.9 & $58.78 ; 1 ;<0.0001$ \\
\hline Citrobacter spp. & 724 & 2.8 & 265 & 1 & 989 & 3.9 & $24.82 ; 1 ;<0.0001$ \\
\hline Streptococci & 623 & 2.4 & 273 & 1.1 & 896 & 3.5 & $8.83 ; 1 ; 0.0296$ \\
\hline Enterobacter spp. & 466 & 1.8 & 357 & 1.4 & 823 & 3.2 & $128.33 ; 1 ;<0.0001$ \\
\hline Proteus spp. & 629 & 2.5 & 147 & 0.6 & 776 & 3 & $121.01 ; 1 ;<0.0001$ \\
\hline Pseudomonas spp. & 306 & 1.2 & 422 & 1.7 & 728 & 2.9 & $11.39 ; 1 ; 0.0007$ \\
\hline Diphtheroids & 309 & 1.2 & 137 & 0.5 & 446 & 1.7 & $145.19 ; 1 ;<0.0001$ \\
\hline Acinetobacter spp. & 54 & 0.2 & 178 & 0.7 & 232 & 0.9 & $2.18 ; 1 ; 0.1400$ \\
\hline S. aureus & 116 & 0.5 & 89 & 0.3 & 205 & 0.8 & - \\
\hline Other Bacteria** & 35 & 0.1 & 27 & 0.1 & 62 & 0.2 & \\
\hline
\end{tabular}

Note: *Non fermentative bacilli (24 strains), Morganella morganii (22 strains), Pantoea spp. (4 strains), Serratia marcescens (4 strains), Hafnia alvei (3 strains), Aeromonas caviae (2 strains), Pasteurella pneumotropica (2 strains) and Providencia spp. (1 strain).

Table 4: Resistance rates of ESBL-producing E. coli strains by years.

\begin{tabular}{|c|c|c|c|c|c|c|c|c|}
\hline \multirow{2}{*}{ Antibiotics } & \multicolumn{2}{|c|}{ All (n: 3,399 } & \multicolumn{2}{|c|}{2018 (n:573) } & \multicolumn{2}{|c|}{ 2014-2017(n:2,826) } & \multirow{2}{*}{$\chi^{2}$} & \multirow{2}{*}{$\begin{array}{l}\text { P value } 2018 \\
\text { vs. 2014- } \\
2017\end{array}$} \\
\hline & $\mathrm{n}^{*}$ & $\%$ & $\mathbf{n}^{*}$ & $\%$ & $\mathrm{n}^{*}$ & $\%$ & & \\
\hline IMP & $15 / 3,393$ & 0.4 & $1 / 571$ & 0.2 & $14 / 2,822$ & 0.5 & 1.112 & 0.2917 \\
\hline MPM & $6 / 832$ & 0.7 & $4 / 571$ & 0.7 & $2 / 261$ & 0.8 & 0.011 & 0.9172 \\
\hline AMK & $171 / 3,392$ & 5 & $25 / 571$ & 4.4 & $146 / 2,821$ & 5.2 & 0.63 & 0.4272 \\
\hline ETP & $158 / 3,046$ & 5.2 & $24 / 571$ & 4.2 & $134 / 2,475$ & 5.4 & 1.384 & 0.2395 \\
\hline
\end{tabular}




\begin{tabular}{|c|c|c|c|c|c|c|c|c|}
\hline TZP & $983 / 3,375$ & 29.1 & $257 / 571$ & 45 & $726 / 2,804$ & 25.9 & 83.99 & $<0.0001$ \\
\hline GEN & $1,049 / 3,255$ & 32.2 & $192 / 570$ & 33.7 & $857 / 2,685$ & 31.9 & 0.672 & 0.4125 \\
\hline CIP & $2,024 / 3,378$ & 59.9 & $398 / 571$ & 69.7 & $1626 / 2,807$ & 57.9 & 27.396 & $<0.0001$ \\
\hline SXT & $2,271 / 3,394$ & 66.9 & $392 / 571$ & 68.7 & $1879 / 2,823$ & 66.6 & 0.938 & 0.3328 \\
\hline SAM & $2,312 / 3,338$ & 69.3 & $322 / 564$ & 57.1 & $1990 / 2,774$ & 71.7 & 47.221 & $<0.0001$ \\
\hline PIP & $2,526 / 2,596$ & 97.3 & - & - & $2526 / 2,596$ & 97.3 & - & - \\
\hline AMP & $1,972 / 2,020$ & 97.6 & - & - & $1972 / 2,020$ & 97.6 & - & - \\
\hline
\end{tabular}

Within the five-year period considered, the most resistance in non-ESBL-producing E. coli strains was found for AMP (70.2\%), SXT (35.2\%), and PIP (32.9). SAM, CIP, CXM, and TZP resistance ranged from 28.8-12.7\%; Resistance to GEN, CPM, CAZ, CTX, CRO, AMK, ETP, MPM, and IMP was below 10\% (between 8.2 and $0.4 \%$ ). Although the resistance rates against IMP, MPM, ETP, CXM, and
SAM were lower in 2018 than in previous years, these low rates were statistically significant only for SAM $(\mathrm{P}<0.0001)$. In 2018, the resistance rates were significantly highly positive, based on the total of the previous four years, for CRO, CTX, CAZ, CPM, GEN, TZP, CIP, and SXT. The details of these results is shown in Table 5.

Table 5: Resistance rates of non-ESBL-producing E. coli strains by years.

\begin{tabular}{|c|c|c|c|c|c|c|c|c|}
\hline \multirow{2}{*}{ Antibiotics } & \multicolumn{2}{|c|}{ All (n: 8,848) } & \multicolumn{2}{|c|}{$2014-2017$ (n: 7,252 ) } & \multicolumn{2}{|c|}{ 2018(n: 1,596) } & \multirow{2}{*}{$\chi^{2}$} & \multirow{2}{*}{$\begin{array}{l}\text { P value } 2018 \\
\text { vs 2014-2017 }\end{array}$} \\
\hline & $\mathbf{n}^{*}$ & $\%$ & $\mathbf{n}^{*}$ & $\%$ & $\mathbf{n}^{*}$ & $\%$ & & \\
\hline IMP & $34 / 8,834$ & 0.4 & $32 / 7,239$ & 0,4 & $2 / 1,595$ & 0,1 & 3.418 & 0.6448 \\
\hline MPM & $11 / 2,323$ & 0.5 & $5 / 729$ & 1,0 & $6 / 1,594$ & 0,3 & 1.017 & 0.3134 \\
\hline ETP & $66 / 7,759$ & 0.9 & $58 / 6,165$ & 0,9 & $8 / 1,594$ & 0,5 & 2.893 & 0.8897 \\
\hline AMK & $113 / 8,105$ & 1.4 & $89 / 6,506$ & 1,4 & $24 / 1,595$ & 1,5 & 0.174 & 0.6765 \\
\hline CRO & $578 / 8,421$ & 6.9 & $414 / 6,826$ & 6,1 & $164 / 1,594$ & 10,3 & 36.055 & $<0.0001$ \\
\hline CTX & $605 / 8,828$ & 6.9 & $423 / 7,231$ & 5,8 & $182 / 1,595$ & 11,4 & 63.289 & $<0.0001$ \\
\hline $\mathrm{CAZ}$ & $658 / 8,842$ & 7.4 & $412 / 7,244$ & 5,7 & $246 / 1,596$ & 15,4 & 179.578 & $<0.0001$ \\
\hline CPM & $717 / 8,834$ & 8.1 & $446 / 7,238$ & 6,2 & $271 / 1,593$ & 17,0 & 206.039 & $<0.0001$ \\
\hline GEN & $668 / 8,127$ & 8.2 & $503 / 6,528$ & 7,7 & $165 / 1,595$ & 10,3 & 11.833 & 0.0006 \\
\hline $\mathrm{TZP}$ & $1,116 / 8,791$ & 12.7 & $540 / 7,195$ & 7,5 & $576 / 1,594$ & 36,1 & 964.897 & $<0.0001$ \\
\hline CXM & $1,165 / 8,065$ & 14.4 & $938 / 6,470$ & 14,5 & $227 / 1,595$ & 14,2 & 0.073 & 0.7869 \\
\hline CIP & $1,789 / 8,799$ & 20.3 & $1,360 / 7,200$ & 18,9 & $429 / 1,596$ & 26,9 & 51.486 & $<0.0001$ \\
\hline SAM & $2,513 / 8,734$ & 28.8 & $2,198 / 7,139$ & 30,8 & $315 / 1,595$ & 19,7 & 77.527 & $<0.0001$ \\
\hline PIP & $2,166 / 6,588$ & 32.9 & $2,166 / 6,588$ & 32,9 & - & - & - & - \\
\hline SXT & $3,096 / 8,792$ & 35.2 & $2,497 / 7,195$ & 34,7 & $599 / 1,596$ & 37,5 & 4.575 & 0.0324 \\
\hline AMP & $3,403 / 4,849$ & 70.2 & $3,403 / 4,849$ & 70,2 & - & - & - & - \\
\hline
\end{tabular}

Note: *The resistant strain/tested strain numbers.

\section{Discussion}

Specimens rejected for microbiological culture and those resulting in contamination lead to economic burdens for the patient and the national economy, while also delaying the specific diagnosis and treatment of a disease. For this reason, laboratory and clinical supervisors must address sampling issues with sufficient sensitivity. In our laboratory, the rate of rejected samples for culture is as low as $0.1 \%$, and this is considered a reasonable result. Mehrotra et al. reported a rate of rejected urine samples in five different hospitals of between 2.4 and $10.0 \%$ and a total of $6.8 \%$ [11]. The results of culture reported as contamination in our laboratory is $10.5 \%$. We consider this an acceptable rate for urine, as urine is one of the best-known specimens for contamination risk. We have used scientific search engines, such as PubMed, Google Scholar and "Web of Science, but we have not yet identified any study that clearly reports rejected sample rates and reported contamination rates for urine cultures. Therefore, our interpretations for these two parameters cannot extend beyond general information.

In the present study, the prevalence of UTI was $15.8 \%$ in Erzurum, which is, to a large extent, representative of the North-eastern Anatolia Region of Turkey. In our region, the prevalence of UTI has shown a significant difference according to age groups, with a rate as high as $23.9 \%$ in children younger than $0-5$ years and $23.5 \%$ in persons aged 65 and over. In these two age groups, the positiv- 
ity was higher in males than in females. The prevalence of UTIs is reported to vary from $12.9 \%$ in the USA to $19.6 \%$ in Europe and $24 \%$ in developing countries [12]. Community-acquired UTIs are a health problem that disproportionately affects women [13]. However, an increase has been noted in the incidence of UTI in infants and in elderly males compared to young adult men. For example, in their meta-analysis, Shaikh et al. reported that the prevalence rates for boys were highest in the first 3 months of life and then decreased; whereas the prevalence rates were highest in the first 12 months in girls [14]. Harrington and Hooton reported that UTIs were more common in women than in men, but the incidence was similar in older men and women [15]. Tanrı̈ver et al. [16] reported an increased UTI incidence in both sexes in persons over 65 years of age, and an almost equal incidence of female/male UTI in the elderly [16]. Elderly women, in particular, experience a loss of lactobacilli, which constitute the dominant protective flora, as a result of decreasing estrogen levels in menopause and therefore have an increased risk of UTI [17]. In addition, the decline in the immune system in old age makes the elderly more susceptible to infection. Our age-related results confirm this classic information on UTIs.

In their meta-analysis study, Beyer et al. reported a prevalence of UTI between 17 and $82 \%$ in eight different countries [18]. The UTI prevalence varies quite extensively across studies, but some studies report much lower and much higher prevalence rates than these rates. For example, Tasbakan et al. [19] in Izmir in Turkey, found an overall prevalence of UTI of $1.82 \%$ [19]. Kant et al. [20] reported a prevalence of UTI varying between 3 and $24 \%$ in pregnant Indian women [20]. Seifu and Gebissa reported a prevalence of UTIs of $90.1 \%$ in their region of Ethiopia [21]. These differences in the prevalence of UTIs are largely due to the co-evaluation of symptomatic or asymptomatic patients in the studies.In our study, E. coli $(48.0 \%)$ had the highest frequency among bacteria isolated from urine, in accordance with the literature. This bacterium was followed by Enterococcus spp., CNS, Klebsiella spp. and Candida spp. strains, in that order. Gram-positive cocci, such as enterococci and staphylococci, are common in both community-acquired and hospital-acquired UTIs. Indeed, Shrestha et al. reported that Enterococcus faecalis from community-based UTIs and S. aureus from catheter-related UTIs were the most common organisms [22].

Urinary candidiasis is the most common fungal nosocomial infection worldwide. Odoki et al. reported that the most commonly isolated uropathogens in the Bushenyi Region of Uganda were $E$. coli (41.9\%), followed by S. aureus and Klebsiella pneumoniae [3]. Seifu and Gebissa reported that E. coli (39.3\%) and Staphylococcus spp. were the most commonly isolated uropathogens in Ethiopia [21,22]. Ahmed et al. [23] in Saudi Arabia, found E. coli and Klebsiella pneumoniae strains as the most common uropathogens [23]. In the context of microorganisms isolated from urine, the results obtained from our study are similar to those of these other studies. In most of the studies, E. coli maintains its primary position, but the same bacteria are found as the next four most common pathogens, although the order changes. Treatment of ESBL-positive E. coli strains that are frequently isolated from community-acquired UTIs is a recognized challenge [24]. More than $1 / 4$ of the $E$. coli strains isolated from urine samples in Erzurum were positive for ESBL production.

A large proportion of ESBL-producing E. coli strains were isolated from women. The most common antibiotic resistance in ESBL-producing E. coli strains was against ampicillin, piperacillin, ampicillin/sulbactam, and trimethoprim-sulfamethoxazole. In ESBL-negative E. coli strains, the highest resistance was observed against ampicillin, trimethoprim-sulfamethoxazole, and piperacillin. For a long time, the rapid increase in antibiotic resistance of $E$. coli strains at the global level has been an issue of grave concern. Edlin et al. [25] found that E. coli was the most common uropathogen in the urine of patients under 18 years of age who were treated in the USA in 2013, and they reported that these strains were the most resistant to ampicillin and TMP-SMX [25]. Lee et al. [26] in 2018, indicated that E. coli resistance to TMP-SMX used in the treatment of UTI had increased significantly [26]. With this trend, they claimed that using this drug worldwide would no longer be possible in the next few years. Ramírez-Castillo et al. [27] found that women and children were the groups most affected by UTIs, mostly infections were community-based, and the most common resistance was to TMP-SMX, ampicillin, and ampicillin-sulbactam [27].

They reported that UTI microorganisms were also frequently resistant to ciprofloxacin, levofloxacin, and cephalosporin. Multidisciplinary studies in European countries (Finland, Germany, Latvia, Poland, Russia, and Sweden), reviewed by Ny et al. found resistance in E. coli isolates produced from urine samples from inpatients aged 18-65 years with uncomplicated UTI symptoms [28]. In a study conducted in Mexico, E. coli had the highest prevalence of ESBL production; a higher overall prevalence of ESBL-producing organisms was reported in nosocomial infections than in community-acquired infections [29]. In that study, ertapenem, imipenem, and amikacin had the highest antibacterial effect. In thirty-one countries, no difference was found between the resistance rates of bacteria isolated from urine samples taken by different methods, including middle urine, catheter, and suprapubic aspiration samples [30].

When we look at the studies related to UTI in our country, E. coli is the most frequently isolated bacterium, both in the community and in inpatients. Yürüyen et al. [31] in their systematic review in Turkey, reported E. coli as the most frequently isolated agent, with a rate of $49 \%$ in inpatients and $70 \%$ in outpatients [31]. The reported E. coli strains were highly resistant to ampicillin, ciprofloxacin, and TMP-SMX. A study conducted in İzmir in 2016 by Yllmaz et al. [31] found that $67 \%$ of the pathogens isolated from UTIs were E. coli strains, and the highest antibiotic resistance was $66.9 \%$ for ampicillin, cefazolin, and cefuroxime [32]. Aktar et al. [33] in their study conducted in Diyarbakır in 2018, reported that 
ESBL-positive E. coli strains had the highest resistance to ampicillin (99.6\%) and the lowest resistance (3.4\%) to meropenem [33]. In that study, the highest resistance in ESBL-negative strains (51.4\%) was seen for ampicillin and the lowest resistance $(0.3 \%)$ was seen for imipenem. In a study published by Kılınçel et al. [34], E. coli was the most commonly isolated agent in patients with diabetes mellitus and obesity, with a rate of $50 \%$; resistance to ampicillin was $85 \%$, to TMP-SMX was $35 \%$, and to gentamicin was $30 \%$; these were the drugs showing the most common resistance [34]. In a study conducted in Ankara in 2015, Süzük et al. [35] found the highest resistance, based on CLSI criteria, against cefuroxime axetil (79.8\%), ampicillin (67.1\%), and ampicillin sulbactam (34.3\%) [35]. According to EUCAST criteria, the highest resistance was reported against ampicillin (63.8\%), TMP-SMX (27.0\%), and ampicillin sulbactam (30.9\%). Topal conducted a study in Mugla and found E. coli as the most common microorganism (69\%) in patients aged $0-15$ years, whereas Klebsiella strains were the most common (48.5\%) pathogen in inpatients [36].

\section{Conclusion}

In conclusion, UTIs are an important health problem in almost all age groups in Erzurum. Health institutions should inform the community about this problem and prevent the spread of UTIs in the local society. The prevalence of community acquired UTIs in young, healthy, and sexually active women points to a need for proper explanation of sexual intercourse and its importance in the spread of UTIs. Imipenem, meropenem, ertapenem and amikacin were found to be the most effective antimicrobials against $E$. coli strains in our region. The resistance of ESBL-positive and -negative E. coli strains to antibiotics used in treatment showed a significant increase in 2018 compared to the previous years. The treatment of UTIs requiring antimicrobial therapy should take into consideration the severity of the disease, as well as the local characteristics of antimicrobial resistance, to ensure administration of the most clinically effective drug at the most appropriate dose and time.

\section{Funding}

None.

\section{Competing Interests}

None declared.

\section{Ethical Approval}

This study was approved by Atatürk University Medical Faculty Clinical Research Ethics Committee (Erzurum, Turkey) on September 19, 2018 (approval no. 06).

\section{References}

1. Bader MS, Loeb M, Brooks AA (2017) An update on the management of urinary tract infections in the era of antimicrobial resistance. Postgrad Med 129(2): 242-258.
2. Shiralizadeh S, Taghizadeh S, Asgharzadeh M, Behrooz S, Pourya G, et al. (2018) Urinary tract infections: raising problem in developing countries. Rev Med Microbiol 29(4): 159-165.

3. Odoki M, Almustapha Aliero A, Tibyangye J, Nyabayo Maniga J, Wampande E, et al. (2019) Prevalence of bacterial urinary tract infections and associated factors among patients attending hospitals in Bushenyi district, Uganda. Int J Microbiol 4246780.

4. Levy I, Ovadia B, Erez E, Rinat S, Ashkenazi S, et al. (2003) Nosocomial infections after cardiac surgery in infants and children: incidence and risk factors. J Hosp Infect 53(2): 111-116.

5. Detweiler K, Mayers D, Fletcher SG (2015) Bacteruria and Urinary Tract Infections in the Elderly. Urol Clin North Am 42(4): 561-568.

6. Umar M, Yaya AA, Yusuf G, Tafinta IY, Aliko AA, et al. (2016) Biochemical characterization and antimicrobial susceptibility trends of Proteus mirabilis isolated from patients suspected with urinary tract infections attending Sickbay Hospital, Zaria, Kaduna, Nigeria. Ann Biol Sci 4(2): $1-8$.

7. O’Keefe LC, Koelle P, Mc Gee Z, Dewberry LS, Wright C, et al. (2019) Innovations in worksite diagnosis of urinary tract infections and the occupational health nurse. Workplace Health Safe 67(6): 268-274.

8. Foxman B (2010) The epidemiology of urinary tract infection. Nat Rev Urol 7(12): 653-660.

9. (2012) Clinical and Laboratory Standards Institute. Performance standards for antimicrobial susceptibility testing. Twenty second informational supplement update. CLSI document M100-S22 32 (3), Clinical and Laboratory Standards Institute, Wayne, PA, USA.

10. (2013) European Committee on Antimicrobial Susceptibility Testing (EUCAST). EUCAST guidelines for detection of resistance mechanisms and specific resistance of clinical and/or epidemiological importance. Version 1.0, December 2013.

11. Mehrotra A, Srivastava K, Bais P (2013) An evaluation of laboratory specimen rejection rate in a North Indian setting-a cross-sectional study. Iosr-Jdms 7(2): 35-39.

12. Medina M, Castillo Pino E (2019) An introduction to the epidemiology and burden of urinary tract infections. Ther Adv Urology 11: 1756287219832172.

13. Nordstrom L, Liu CM, Price LB (2013) Foodborne urinary tract infections: a new paradigm for antimicrobial-resistant foodborne illness. Front Microbiol 4: 29.

14. Shaikh N, Morone NE, Bost JE, Farrell MH (2008) Prevalence of urinary tract infection in childhood: a meta-analysis. Pediatr Infect Dis J 27(4): 302-308.

15. Harrington RD, Hooton TM (2000) Urinary tract infection risk factors and gender. J Gend Specif Med 3(8): 27-34.

16. Tanrı̈ver Ö, Tezvaran Z, Ülgen A (1998) Urinary tract infections in elderly patient, evaluation, treatment and prevention. J Turkish Fam Physician 2(2): 58-64.

17. Rowe TA, Juthani Mehta M (2013) Urinary tract infection in older adults. Aging health 9(5).

18. Wilson ML, Gaido L (2004) Laboratory diagnosis of urinary tract Infections in adult patients. Clin Infect Dis 38(8): 1150-1158.

19. Isikgoz Tasbakan M, Durusoy R, Pullukcu H, Sipahi OR, Ulusoy S (2013) Turkish Nosocomial Urinary Tract Infection Study Group. Hospitalacquired urinary tract infection point prevalence in Turkey: differences in risk factors among patient groups. Ann Clin Microbiol Antimicrob 12: 31.

20. Kant S, Lohiya A, Kapil A, Gupta SK (2017) Urinary tract infection among pregnant women at a secondary level hospital in Northern India. Indian J Public Health 61(2): 118-123. 
21. Seifu WD, Gebissa AD (2018) Prevalence and antibiotic susceptibility of Uropathogens from cases of urinary tract infections (UTI) in Shashemene referral hospital, Ethiopia. BMC Infect Dis 18(1): 30.

22. Shrestha LB, Baral R, Khanal B (2019) Comparative study of antimicrobial resistance and biofilm formation among Gram-positive uropathogens isolated from community-acquired urinary tract infections and catheterassociated urinary tract infections. Infect Drug Resist 12: 957-963.

23. Ahmed SS, Shariq A, Alsalloom AA, Babikir IH, Alhomoud BN (2019) Uropathogens and their antimicrobial resistance patterns: Relationship with urinary tract infections. Int J Health Sci (Qassim) 13(2): 48-55.

24. Hertz FB, Schønning K, Rasmussen SC, Littauer P, Knudsen JD, et al. (2016) Epidemiological factors associated with ESBL- and non ESBLproducing E. coli causing urinary tract infection in general practice. Infect Dis (Lond) 48(3): 241-245.

25. Edlin RS, Shapiro DJ, Hersh AL, Copp HL (2013) Antibiotic resistance patterns of outpatient pediatric urinary tract infections. J Urol 190(1): 222-227.

26. Lee DS, Lee SJ, Choe HS (2018) Community-acquired urinary tract infection by Escherichia coli in the era of antibiotic resistance. Biomed Res Int 7656752

27. Ramírez Castillo FY, Moreno Flores AC, Avelar González FJ, Márquez Díaz F, Harel J, et al. (2018) An evaluation of multidrug-resistant Escherichia coli isolates in urinary tract infections from Aguascalientes, Mexico: cross-sectional study. Ann Clin Microbiol Antimicrob 17(1): 34.

28. Ny S, Edquist P, Dumpis U, Gröndahl Yli Hannuksela K, Hermes J, et al. (2019) Antimicrobial resistance of Escherichia coli isolates from outpatient urinary tract infections in women in six European countries including Russia. J Glob Antimicrob Resist 17: 25-34.

29. Ponce de Leon A, Rodríguez Noriega E, Morfín Otero R (2018) Antimicrobial susceptibility of gram-negative bacilli isolated from intra-

ISSN: 2574-1241

DOI: 10.26717/BJSTR.2020.24.004029

Osman Aktaş. Biomed J Sci \& Tech Res

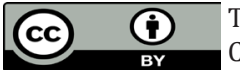

This work is licensed under Creative Commons Attribution 4.0 License

Submission Link: https://biomedres.us/submit-manuscript.php abdominal and urinary-tract infections in Mexico from 2009 to 2015: Results from the Study for Monitoring Antimicrobial Resistance Trends (SMART). PLoS One 13(6): e0198621.

30. Bryce A, Hay AD, Lane IF, Thornton HV, Wootton M, et al. (2016) Global prevalence of antibiotic resistance in paediatric urinary tract infections caused by Escherichia coli and association with routine use of antibiotics in primary care: systematic review and meta-analysis. British Med J 352: i939.

31. Yürüyen C, Gürol Y, Kaleağasioğlu SF, Kaspar EÇ, Yilmaz G (2017) Isolation rates and antibiotic susceptibilities of different Enterobacteriaceae species as urinary tract infection agents in Turkey: a systematic review. Turkish J Med Sci 47(3): 979-986

32. Yılmaz N, Ağuș N, Bayram A, Samlıŏlu P, Sirin MC, et al. (2016) Antimicrobial susceptibilities of Escherichia coli isolates as agents of community-acquired urinary tract infection (2008-2014). Turk J Urol 42(1): 32-36

33. Aktar GS, Ayaydın Z, Onur AR, Vural DG, Temiz H (2018) Resistance rates against various antimicrobials in Escherichia Coli strains isolated from urine samples. Kocaeli Tip Derg 7(1): 8-13.

34. Kılınçel Ö, Öztürk CE, Ankaralı H, Bolu S, Önder E, et al. (2018) Urinary system infection at diabetes mellitus and obesity patients: A retrospective evaluation. Düzce Med J 20(2): 41-45.

35. Süzük, S, Kașkatepe B, Avcıküçük H, Aksaray S, Bașustaoğlu A (2015) The comparison of antibiotic susceptibilities of uropathogenic Escherichia coli isolates in transition from CLSI to EUCAST. Mikrobiyol Bul 49(4): 494-501.

36. Topal Y (2018) Urinary tract infection in children: an assessment between current data. Ortadogu Tip Derg 10(1): 26-33.

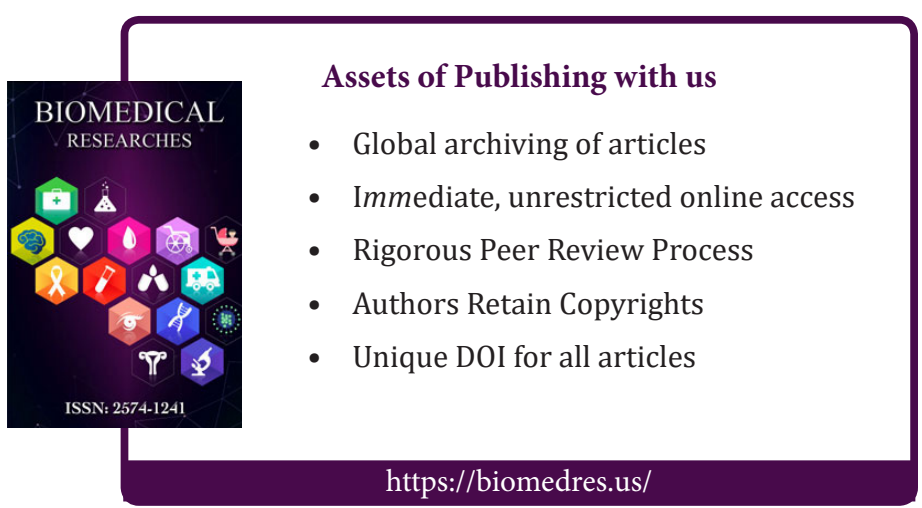

